\title{
Hysteresis analysis of a notched continuum manipulator diriven by tendon
}

\author{
Hong-Jian Yu ${ }^{1}{ }^{*}$, Wen-Long Yang ${ }^{1, *}$, Zheng-Xin Yang ${ }^{1}$, Wei Dong ${ }^{1}$, Zhi-Jiang Du ${ }^{1}$, and Zhi-Yuan Yan ${ }^{1}$ \\ ${ }^{1}$ State Key Laboratory of Robotics and System, Harbin Institute of Technology (HIT), Harbin, China \\ * These authors contributed equally to this work. \\ Correspondence: Zhi-Yuan Yan (zhiyuanyan@163.com)
}

Received: 28 September 2017 - Revised: 6 April 2018 - Accepted: 17 May 2018 - Published: 8 June 2018

\begin{abstract}
Continuum manipulators are widely used in minimally invasive surgical robot systems (MISRS) because of their flexibility and compliance, while their modelling and control are relatively difficult and complex. This paper proposes an improved hysteresis model of a notched continuum manipulator based on the classical Bouc-Wen model, which can reduce errors and increase the accuracy of the kinematic-mechanics coupled model. Then parameters are identified by the mean of genetic algorithm (GA). Hysteresis phenomenon of the mentioned manipulator is actually caused by many factors such as the hysteresis property of Hyperelastic Nitinol Alloy (HNA), the elastic deformation of tendon and the friction between the tendon and the tube. The results of both static and dynamic experiments show that the introduced hysteresis model can eliminate the positional difference between forward and reverse bending processes, and thus improve the forecast precision of deformation during motion. This model can also be used to compensate modelling errors caused by hysteresis of other similar systems.
\end{abstract}

\section{Introduction}

Continuum robots, different from conventional discrete joints robots, do not contain rigid links and identifiably rotational joints, whose deformation bases on intrinsic elastic and compliant character (Robinson and Davies, 1999). Because of their small volume, light weight, flexible movement and compliant interaction, continuum manipulators are widely used in MISRS (Burgner-Kahrs et al., 2015). In the past twenty years, lots of novel and useful continuum manipulators were reported. Simaan and Taylor (2004) designed a tendon-driven continuum manipulator which contains several elastic continuum backbones and spacer disks. Webster et al. (2007) and Dupont et al. (2010) presented a concentric tube manipulator comprising several nested pre-curved tubes. $\mathrm{Li}$ and $\mathrm{Du}$ (2013) proposed a continuum manipulator which consists of multiple section set backbones composed of short links. In addition, notching different type incisions on a hyper elastic tube was also utilized to generate continuum manipulators (Du et al., 2015a; Gao et al., 2017). The modelling for continuum manipulators is a challenge in academia because of their special deformation characterises.
A variety of modelling methods were developed which can be divided into two groups (Walker, 2013). Some researchers adopted overall modelling to map driving force and deformation using diverse theorems: energy method such as the principle of virtue power and the transformation of potential energy (Tatlicioglu et al., 2007; Rone and Ben-Tzvi, 2014), Cosserat rod theory (Jones et al. 2009; Rucker and Iii, 2011; Giorelli et al., 2012), constant curvature assumption (Camarillo et al., 2008; York et al., 2015; Wu et al., 2017), etc. Moreover, some researchers established a unit model first and then built an integrate deformation model considering unit kinematics and mechanics character (Du et al., 2015b; Kato et al., 2015).

However, the hysteresis of the continuum manipulator attracts less attention compared with the mechanics and kinematics. Actually, the hysteresis phenomenon widely exists in the nature which affects control accuracy or even stability of most mechanical systems seriously, so many researches are conducted on this phenomenon (Liu et al., 2014). Seyfferth et al. (1995) built a mechanical model for the harmonic drive robotic transmissions considering compliance, 
friction and hysteresis. Kwok et al. (2007) established a hysteresis model based on hyperbolic tangent non-linear function to improve the model precision of magnetorheological fluid dampers. Van Damme et al. (2008) proposed a Preisach based hysteresis model to solve the specific shape of the force-contraction characteristic of pneumatic muscles. Ruderman et al. (2009) designed a novel approach which combines distributed Preisach model and generalized MaxwellSlip friction model to explore elastic robot joints with hysteresis and backlash. Xiao and Guo (2010) conducted a research on modelling and parameter identification of the hysteresis existed in the micromanipulator for human scale teleoperation system. Xu (2013) presented an approach to realizing hysteresis identification and compensation of piezoelectric actuators using least squares support vector machine based hysteresis model. Miyasaka et al. (2016) proposed a hysteresis model for longitudinally loaded cables which can be applied for control of cable driven systems such as the RAVEN II surgical robotic research platform.

Most of the kinematics modelling of continuum manipulators ignored hysteresis problems, Kang et al. (2013) demonstrated the sigmoid shape in length versus pressure curve caused by hysteresis when model pneumatically actuated continuum manipulator, but they ignored this phenomenon in order to simply the model. Kato et al. (2016) took hysteresis of a tendon-driven continuum robot for neuroendoscopy into account by adding friction coefficient into their model instead of using fitting parameters, and this research only worked out the hysteresis caused by the friction. However, the hysteresis effect of our proposed continuum manipulator has many factors, including the hysteresis property of HNA and the elastic deformation of tendon (CruzHernandez and Hayward, 1998), as well as the friction and backlash happened in the tendon transmission (Swevers et al., 2000). Therefore, consideration of hysteresis problem of the notched continuum manipulator driven by the tendon during modelling is important to improve the modelling accuracy, and also the designed hysteresis model and parameter identification method in this paper is effective.

Our previous work designed and modelled a notched continuum manipulator driven by tendons (Du et al., 2015b). This paper gives a further research, which mainly focuses on solving the nonlinear position difference caused by hysteresis character of the continuum manipulator between forward and reverse bending processes under the same tendon displacement. A brief review of the overall structure, mechanics model and kinematics model of the notched continuum manipulator is introduced in Sect. 2. In Sect. 3, a theoretical analysis based on experimental results is presented, and then a hysteresis model of the continuum manipulator based on the classical Bouc-Wen model is established. Also, the unknown parameters are identified by GA in this section. A prototype is machined and the experiment platform is constructed, and then static and dynamic experiments are con-

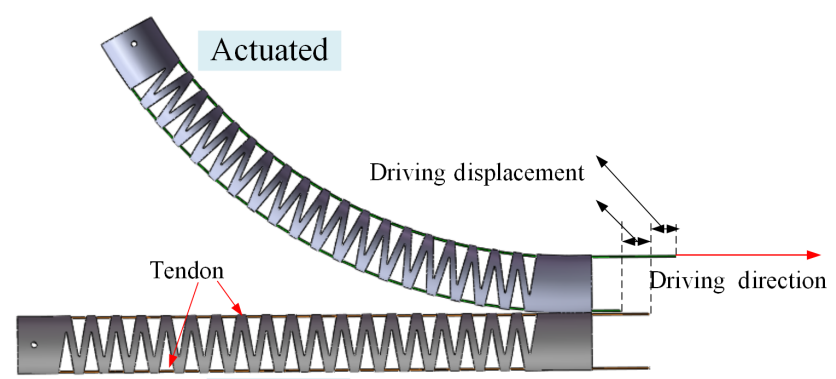

Intialize

Figure 1. Schematic projection of the notched continuum manipulator.

ducted in Sect. 4 to verify the effectiveness of the improved hysteresis model. Finally, this paper is summarized in Sect. 5.

\section{Kinematics model of the notched continuum manipulator}

This section reviews the previous contributions which plays a fundamental role in the subsequent research, including the overall structure, mechanics model and kinematics model of a single notched ring (SNR) unit and the integrated manipulator (Du et al., 2015b).

\subsection{Structure of the notched continuum manipulator}

The schematic projection of the notched continuum manipulator is shown in Fig. 1. The overall structure is made of HNA tube on which several V-shape cuts are processed by lowspeed wire cut electrical discharge machining (LS-WEDM), and two tendons are fixed at the tip of the manipulator to transmit driving force. The compliant character is guaranteed by intrinsic super-elasticity of the material. The sufficient inner cavity can also provide enough volume for tendons of other joints as well as signal wires. The continuum manipulator can be considered as an integrated structure with several SNRs. This design ensures relatively small volume, easy processing, long life and good biocompatibility. In addition, the symmetrical notches can supply bending capability in a specific direction and guarantee enough stiffness in other directions so that it improves the stability of the continuum manipulator.

\subsection{Kinematics model of the notched continuum manipulator}

The proposed continuum manipulator is driven by tendons, whose total deformation is integrated by all SNRs' deformation. Since kinematics and mechanics are coupled in the proposed continuum manipulator, our previous work developed an innovative model describing the manipulator deformation from "micro" to "macro". A SNR is presented in Fig. 2 which 


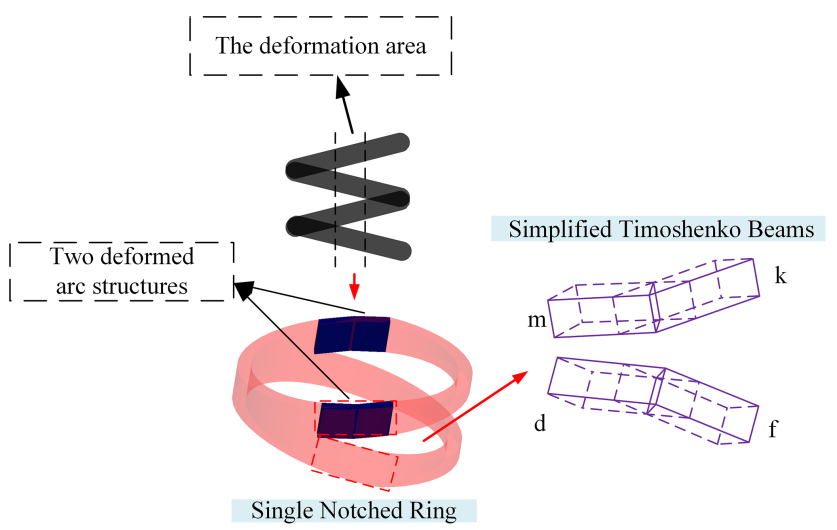

Figure 2. Simplified Timoshenko beams model of a SNR.

is divided into deformed and un-deformed regions. In the 3$\mathrm{D}$ space, for each ring in the SNR, there are two symmetrical deformed arc structures with small central angle, i.e., the main deformation area concentrates on these arc structures with small central angle. Then each deformation arc is simplified into two 3-D Timoshenko beam elements with three nodes as shown in Fig. 2. Finally, the mechanics model is concentrated on the simplified Timoshenko beam elements, which is built based on Timoshenko beam theory (Du et al., 2015b).

Based on the mechanics, the relationship between the deformation of the SNR, the deformation of continuum manipulator and the driving force can be expressed as follows,

$\theta_{\text {sum }}=\sum_{i=1}^{N} \theta_{\mathrm{kd}}(i)=\sum_{i=1}^{N}\left(\theta_{\mathrm{k}}(i)+\theta_{\mathrm{d}}(i)\right)=g\left(F_{\mathrm{T}}\right)$

where $\theta_{\text {sum }}$ is the total deformation angle of the continuum manipulator, $i$ is the order of SNRs, $F_{\mathrm{T}}$ is the driving force, $N$ is the number of SNRs, $\theta_{\mathrm{d}}(i)$ is the deformation angle at node $\mathrm{d}$ of $i$ th SNR, $\theta_{\mathrm{k}}(i)$ is the deformation angle at node $\mathrm{k}$ of $i$ th SNR, and $\theta_{\mathrm{kd}}(i)$ is the angle deformation of the $i$ th SNR. Based on the deformation angles $\theta_{\mathrm{d}}(i), \theta_{\mathrm{k}}(i)$ and $\theta_{\mathrm{kd}}(i)$ derived from the mechanics model, the total deformation angle $\theta_{\text {sum }}$ can be calculated.

Besides, an equivalent kinematics model of the continuum manipulator based on mechanics model is proposed. The continuum manipulator is decomposed into several equivalent rigid links and discrete joints. The equivalent kinematics coordinate system is established as shown in Fig. 3.

When the continuum manipulator is equivalent to the series connection of discrete joints, the forward kinematics model can be built via Denavit-Hartenberg (D-H) method,

$$
\begin{aligned}
\mathbf{T}_{0}^{N} & =\mathbf{T}_{b}^{0} \cdot \mathbf{T}_{0}^{1}(H(1), \theta(1)) \cdot \mathbf{T}_{1}^{2}(H(2), \theta(2)) \\
& \cdots \mathbf{T}_{i-1}^{i}(H(i), \theta(i)) \cdots \mathbf{T}_{N-1}^{N}(H(N), \theta(N)) \cdot \mathbf{T}_{N}^{d}
\end{aligned}
$$

where $\mathbf{T}_{b}^{0}$ and $\mathbf{T}_{N}^{d}$ are related to the choice of coordination system and structure parameters, $\mathbf{T}_{i-1}^{i}$ is the transformation

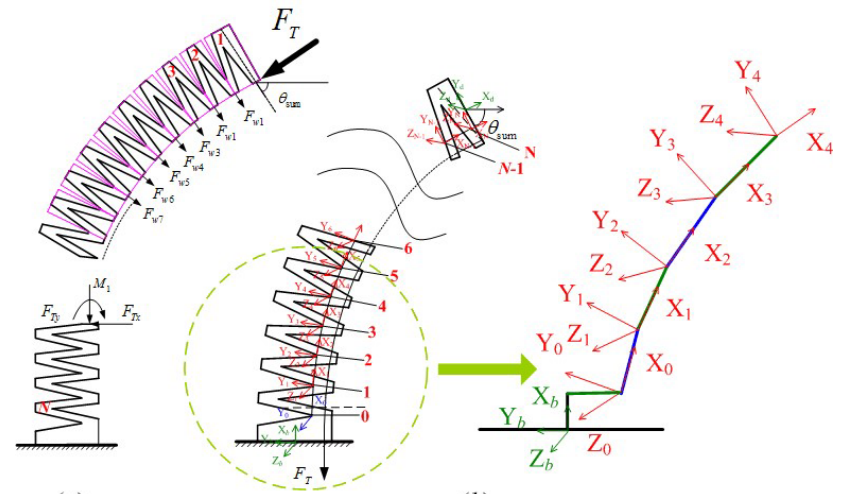

(a)

(b)

Figure 3. Equivalent kinematics coordination system of the notched continuum manipulator.

matrix for the $i$ th equivalent joint, $H(i)$ and $\theta(i)$ are the equivalent link lengths and joint angles, respectively. All the parameters in Eq. (2) can be obtained according to the previous mechanics model (Du et al., 2015b).

In addition, due to the redundancy of the continuum manipulator, it is complicated to obtain explicit expression of angle deformation when the distal position of the manipulator is given. The curve fitting method is used to solve the inverse kinematics. Finally, the relationship between driving space (displacement of tendon), joint space (deformation angle of the manipulator) and Cartesian space (distal position) is finally established,

$x=\xi\left(\theta_{\text {sum }}\right)=\zeta\left(P_{x}(x), P_{y}(x), P_{z}(x)\right)$

where $x$ is the displacement of tendon, which can be expressed as the function $\xi$ of the deformation angle of the continuum manipulator $\left(\theta_{\text {sum }}\right)$ and the function $\zeta$ of the distal positions in Cartesian space of the continuum manipulator $\left(P_{x}(x), P_{y}(y), P_{z}(z)\right)$ are respectively (Du et al., 2015b).

\section{Hysteresis modelling of the notched continuum manipulator}

The hysteresis characteristic of HNA and the elastic deformation of tendon generate the hysteresis characteristics of the continuum manipulator. This section concentrates on the nonlinear deformation characteristic caused by the hysteresis effect. The hysteresis effect of the system is analysed firstly, and then a hysteresis model of the manipulator is established based on the classical Bouc-Wen model. Finally, the parameters of the hysteresis model are identified.

\subsection{Analysis of hysteresis effect of the notched continuum manipulator}

To explore the systematic hysteresis character of the continuum manipulator, static and dynamic experiments are implemented and the experiment setup will be discussed in Sect. 4 . 


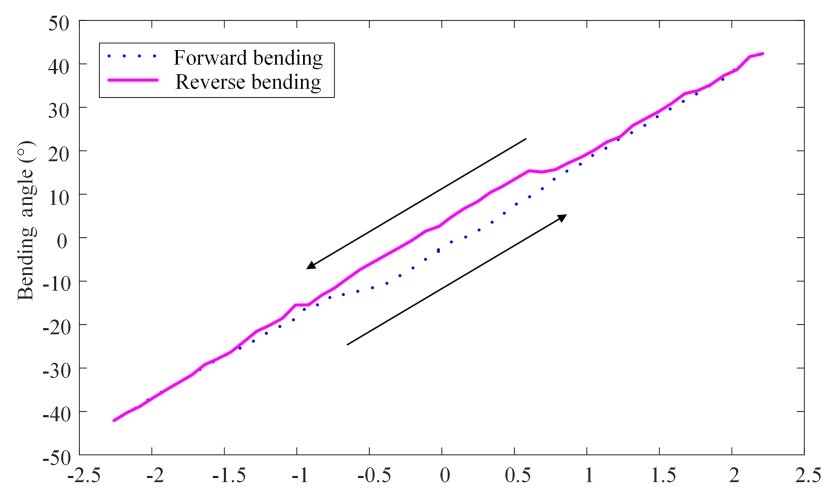

(a) $10 \mathrm{~N}$ preload

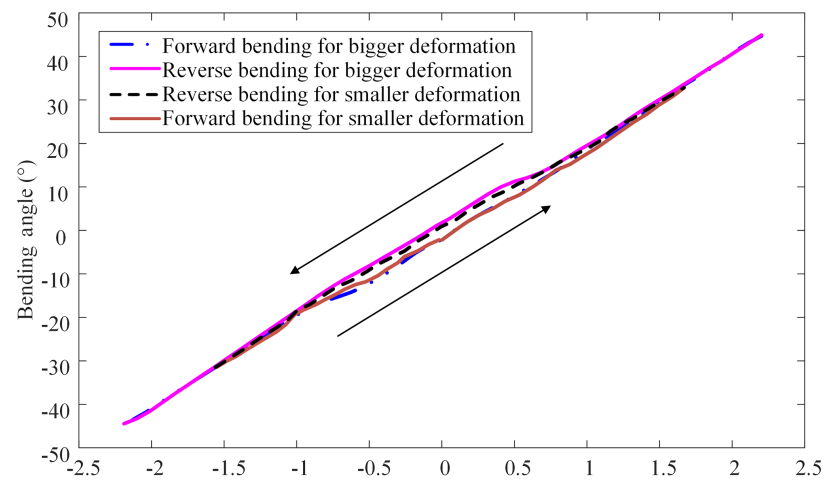

(b) $20 \mathrm{~N}$ preload

Figure 4. Static experimental results under discrete signal.

The main factors that influence the hysteresis effect of the manipulator includes: the elasticity and backlash of tendon, the friction force between the tendon and the manipulator, and the hysteresis behaviour of HNA.

Figure 4 is the results of the static experiment when the continuum manipulator is actuated at regular $0.1 \mathrm{~mm}$ displacement intervals. Figure $4 a$ and $b$ show the manipulator displacements under the preload of 10 and $20 \mathrm{~N}$ respectively. During the experiments, the motor is actuated in position mode, in which the actuated displacement starts from $0 \mathrm{~mm}$ and then reached 2.2, $0,-2.2$ and $0 \mathrm{~mm}$ in sequence. The continuum manipulator is driven by two tendons via actuating the motor clockwise and anticlockwise. It can be seen that the forward and reverse curves do not coincide exactly and present the obvious hysteresis, in which one is below the theoretical bending angle curve and the other one is above it. Based on the results with different preloads, it can be found that the magnitude of preload force effects systematic hysteresis character. The hysteresis is less distinct when the manipulator is actuated to bigger bending angle. The maximum hysteresis displacements under the preload of 10 and $20 \mathrm{~N}$ are 3.52 and $2.21^{\circ}$ respectively. A reasonable larger preload force means a less obvious hysteresis. In addition, the backlash and elasticity of tendon are reduced as a result of a larger preload, and more smooth and stable movement is achieved under larger preload. According to Fig. 4b, with the same

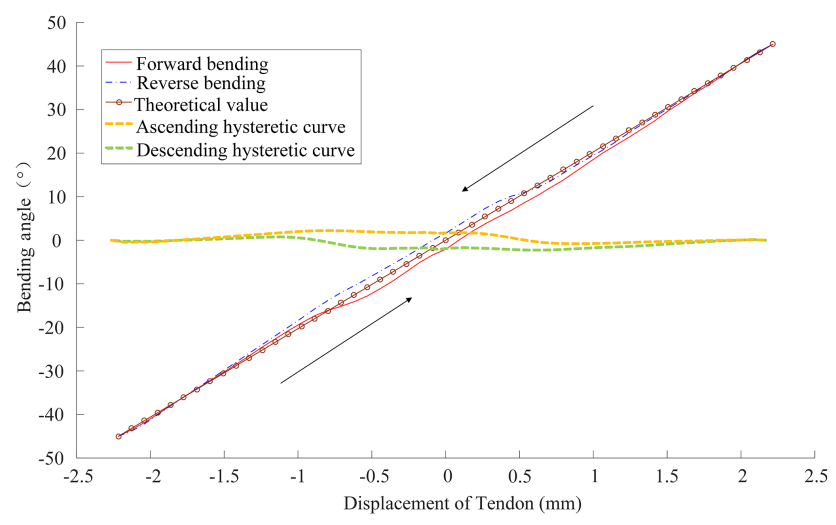

Figure 5. Hysteresis loop of the notched continuum manipulator.

preload, the hysteresis is approximately the same when maximum deformation angle is increased so the proposed hysteresis model can be used in practical application.

A further analysis of the experimental results under $20 \mathrm{~N}$ preload with bigger maximum deformation is shown in Fig. 5. There are three kinds of angle in Fig. 5, i.e., the experimental bending angle, the theoretical bending angle calculated by the aforementioned kinematics model and the hysteresis angle by computing the difference between experimental and theoretical bending angle. The hysteresis loop indicates that the bending angle of continuum manipulator in a load cycle has obvious hysteresis phenomenon. The most insignificant hysteresis happens under the maximum driving force, for in this region, the deformation of the manipulator not only relies on the tension of tendon, but the spring back caused by intrinsic elasticity also provides recovery capacity. On the other hand, the change of bending direction mainly depends on tension applied by the other side tendon, which is the reason why the most significant hysteresis happens under the minimum driving force, and in this case, both the elasticity and backlash of tendon can cause hysteresis.

Figure 6 shows the results of the dynamic experiment, in which the bending angle curve is described in the time domain. An obvious difference exists between the theoretical deformation and the actual deformation. The HNA hysteresis, friction force, tendon elasticity and other effects that generate the difference, in which the manipulator cannot reach the peak theoretical value due to the hysteresis accumulation under sinusoidal driving signal. Besides, when the manipulator is actuated continuously, the time delay happens between actual deformation and theoretical deformation.

\subsection{Hysteresis model of the notched continuum manipulator based on the Bouc-Wen model}

The static and dynamic experimental results show that hysteresis phenomenon does exist in the movement of continuum manipulator. To improve the modelling precision and reduce the hysteresis disturbance, the hysteresis compensa- 


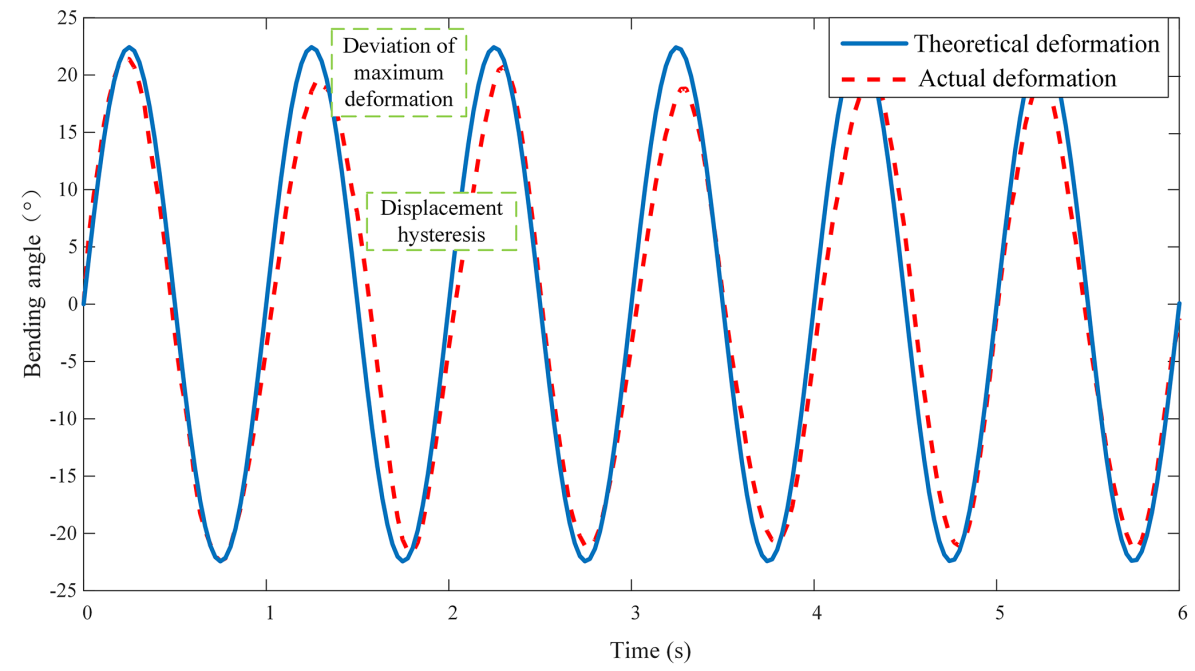

Figure 6. Dynamic experimental results under sinusoidal signal.

tion is necessary. In this section, a hysteresis model is developed to compensate the errors in the forward and reverse bending.

The classical Bouc-Wen algorithm model uses a nonlinear differential equation with uncertain parameters to describe the systematic hysteresis character. By selecting different model parameters, the hysteresis curve of various shapes can be simulated, including viscous stage and slip stage (Smyth et al., 2002). This method has the advantages of few parameters, simple format and convenient application, which is widely used in the modelling and control of hysteresis structure in engineering field (Ismail et al., 2009).

The Bouc-Wen model is composed of a hysteretic system, a spring and a damper in parallel as shown in Fig. 7, whose mechanical property can be expressed as (Wen, 1976),

$F(x, z)=c \dot{x}+k\left(x-x_{0}\right)+\alpha z$

where $F$ is the nonlinear restoring force of the system, $k$ is the stiffness coefficient, $\alpha$ is the post-yield to pre-yield stiffness ratio, $x$ is the displacement, $\dot{x}$ is the velocity, $x_{0}$ is the initial deformation of the spring, $z$ is the hysteresis restoring variable and is expressed via a differential Eq. (5) composed of four parameters: $\beta, \gamma$ and $n$ determine the hysteresis shape, and $A$ defines the tangent stiffness,

$\dot{z}=A \dot{x}-\beta|\dot{x}||z|^{n-1} z-\gamma \dot{x}|z|^{n}$

where $\dot{z}$ is the first derivative of the hysteresis displacement.

The Eq. (4) can be considered as the sum of linear stiffness component and nonlinear hysteresis components. Based on the Eq. (5), once the parameters $A, \beta, \gamma$ and $n$ are determined, the hysteresis components can be derived by solving the differential equation of Eq. (5).

The classical Bouc-Wen model given above is used to describe the hysteresis relationship between the restoring force

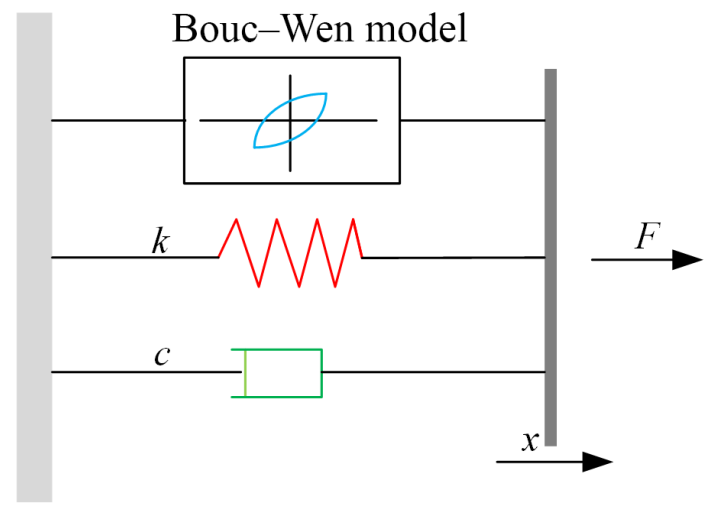

Figure 7. Diagram of the Bouc-Wen model.

and the displacement of the nonlinear system, which divides the mechanical properties into the systemic theoretical movement component $c \dot{x}+k\left(x-x_{0}\right)$ and the nonlinear hysteresis component $\alpha z$. Based on the idea of the Bouc-Wen model, this paper decomposes the hysteresis curve of continuum manipulator into the theoretical nonlinear kinematic component and nonlinear hysteresis component. The improved hysteresis model of the continuum manipulator can be expressed as,

$\theta(x)=f(x)+h(x)$

where $\theta(x)$ is the actual deformation angle of the continuum manipulator, $f(x)$ is the nonlinear deformation angle of the continuum manipulator in joint space based on the kinematic-mechanics coupled model, which is already obtained in the previous section, $h(x)$ is the nonlinear hysteresis displacement of the continuum manipulator, and $x$ is the driving displacement of tendon. 
According to the Bouc-Wen model given by Eq. (5), the expression $h(x)$ of the hysteresis term in Eq. (6) can be expressed as a differential equation,

$\dot{h}(x)=A \dot{f}(x)-\beta|\dot{f}(x)||h(x)|^{n-1} h(x)-\gamma \dot{f}(x)|h(x)|^{n}$

where $\dot{h}(x)$ and $\dot{f}(x)$ are the differential expressions of the nonlinear deformation angle $f(x)$ and the nonlinear hysteresis displacement $h(x)$ of the continuum manipulator, $A, \beta, \gamma$, $n$ are the parameters to be identified.

According to the kinematics and the hysteresis models in Eqs. (6) and (7), four unknown parameters $(A, \beta, \gamma, n)$ need to be determined to get a certain hysteresis model of the continuum manipulator, so a more accurate relationship between the tendon displacement and the bending angle can be obtained.

\subsection{Parameters identification of the hysteresis model}

This paper uses the genetic algorithm (GA) to identify the parameters of the nonlinear hysteresis model which is good at solving multivariable optimization and global optimization problem (Tavakolpour et al., 2010). The method can easily and effectively identify the unknown parameters in the hysteresis model based on the data obtained in the static experiment. To identify the parameters of the hysteresis model, two steps are necessary. Firstly, the fitness function should be defined. Secondly, the GA toolbox parameters including the fit function number of variables, initial population, generation, stall generation, and function tolerance should be defined.

Step 1: The theoretical bending angle $\hat{\theta}$ of the continuum manipulator is calculated by the kinematic-mechanics coupled model. The actual bending angle $\theta$ of the continuum manipulator is measured by experiment. Then the fitness function is designed as follows,

fitnessfunc $=\frac{1}{m} \sum_{i=1}^{m}\left(\frac{\hat{\theta}_{i}-\theta_{i}}{\theta_{\max }-\theta_{\min }}\right)$

where $m$ is the number of data points, $\theta_{\max }$ is the maximum bending angle, and $\theta_{\min }$ is the minimum bending angle.

Step 2: The GA toolbox parameters are defined as follows.

Fit function: @fitnessfunc;

Number of variables: 4;

Initial population: [1000, 1000, 1000, 1000];

Generation: 1000;

Stall generation: inf;

Function tolerance: $1 \times 10^{-6}$.
According to the static experiment which gives the corresponding bending angle $\theta(t)$ nder the displacement of tendon $x(t),(A, \beta, \gamma, n)$ in the hysteresis model can be identified, therefore Eq. (7) is determined. Then the theoretical value of the hysteresis component can be computed using fourth order Runge-Kutta method (Hairer et al., 2006). The continuum manipulator used in this paper has 14 SNRs. The actuator servo-controls the motor by step of 1000 pulses, and the control signal is expressed as follows.

$s(t)=\left\{\begin{aligned}-1000 t+25000 & {[0,50] } \\ 1000 t-75000 & {[50,100] }\end{aligned}\right.$

The displacement of tendon and the actual bending angle of the continuum manipulator corresponding to the control signal $s(t)$ are shown in Figs. 5 and 6 respectively. The parameters of the hysteresis model are determined using the sample in the static experiment by off-line identification, and identified values are shown as follows.

$A=1.895, \beta=0.315, \gamma=0.2606, n=1.176$

\section{Experimental results and data analysis}

This section explores the hysteresis model of the continuum manipulator by comparing the improved model with the experiment results, and both the static and dynamic experiments are employed to verify the hysteresis model.

\subsection{Establishment of experiment platform}

The experiment platform is shown in Fig. 8. For each tendon, one side is fixed at the tip of the continuum manipulator, and the other side is rotated around and fixed on an output wheel. Two wheels are driven by one motor and are installed on the output shaft in the opposite direction. The displacement of tendons is feedback by the digital encoder integrated on the motor. The image of the continuum manipulator is taken by the GigE Industrial CCD camera produced by Point Gery Company in Canada, and MATLAB is involved in processing images. The side wall profiles (including the endpoint positions) of the continuum manipulator are extracted firstly, and then the bending angles in different positions can be calculated.

\subsection{Static experimental results}

In static experiment, data are collected point by point, the displacement of tendon changes from -2.2 to $2.2 \mathrm{~mm}$ then back to $-2.2 \mathrm{~mm}$ in a discrete signal cycle with a step of $0.1 \mathrm{~mm}$, the corresponding bending angle of the continuum manipulator changes from -45 to $45^{\circ}$ and back to $-45^{\circ}$. When the displacement of tendon is negative, the opposite site tendon provides driving tension. When the displacement of tendon is positive, the objective tendon provides driving tension. Ten 


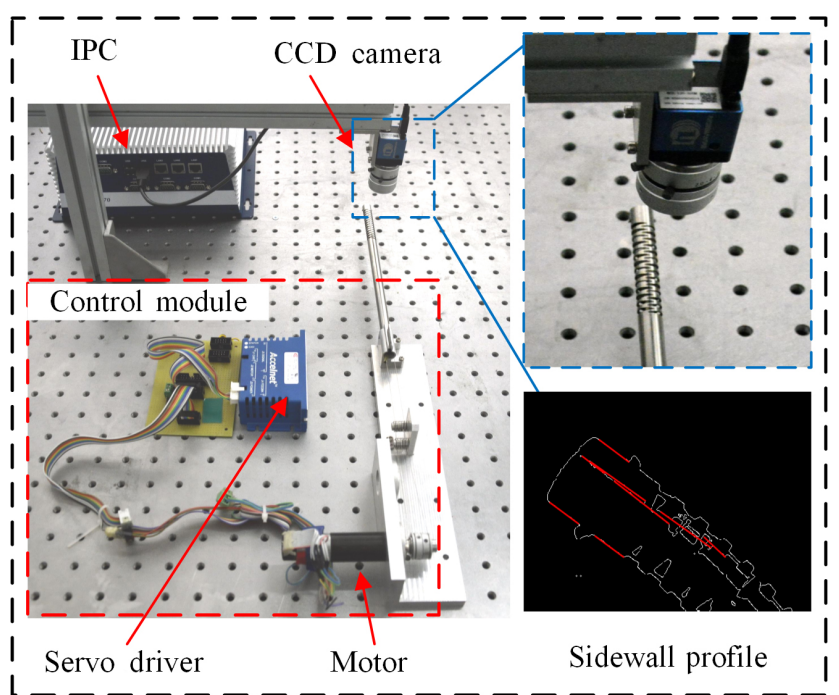

Figure 8. Experiment platform for the hysteresis model of the notched continuum manipulator.

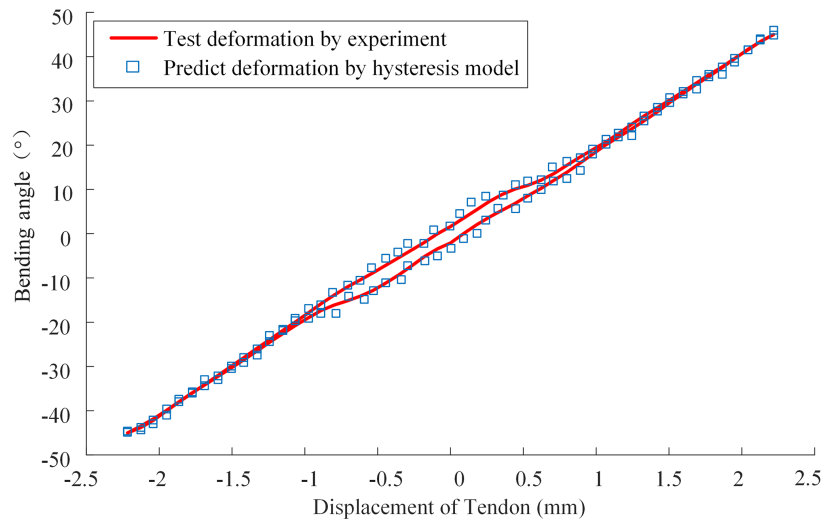

Figure 9. Comparison between actual and theoretical bending angle under discrete signals.

cycles are completed in each test and the actual bending angle is determined by averaging ten times measurements.

The hysteresis model of the continuum manipulator is obtained by putting the identified parameter of Eq. (10) back into Eq. (7), so that an improved hysteresis model is obtained. By using the fourth order Runge-Kutta method, the compensated theoretical bending angle of the continuum manipulator under the displacement $x(t)$ is obtained. In Fig. 9, the red line is the actual bending angle measured in the experiment, while the blue square is the predicted bending angle calculated by the improved hysteresis model which takes hysteresis characteristic into consideration under different load direction. Compared with the previous kinematic-mechanics coupled model, the improved hysteresis model can predict bending angle according to the tendon displacement and the load direction, which reflects authentic deformation and therefore can predict the bending angle more exactly.

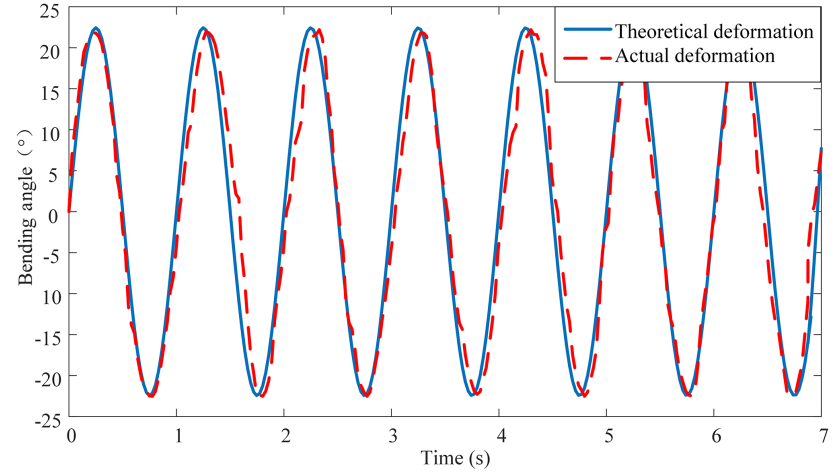

Figure 10. Comparison between actual and theoretical bending angle under sinusoidal signals.

Table 1. Errors analysis of the improved hysteresis model.

\begin{tabular}{lrrr}
\hline Driving signal & \multicolumn{3}{c}{ Errors } \\
\cline { 2 - 4 } & $\Delta_{\max }^{\theta}$ & $\delta_{\max }^{\theta}$ & $\delta$ \\
\hline Discrete signal & $1.48^{\circ}$ & $3.23 \%$ & 0.0133 \\
Sinusoidal signal & $1.61^{\circ}$ & $3.58 \%$ & 0.0162 \\
\hline
\end{tabular}

\subsection{Dynamic experimental results}

In the dynamic experiment, the driving signal is set as a $1 \mathrm{~Hz}$ sinusoidal signal with peak value of 25000 pulses, and the similar measurement as before is employed to measure the actual bending angle. Figure 10 shows the corresponding experimental results and theoretical calculation after the hysteresis model is added. Compared with Fig. 6, the maximum deviation and the displacement hysteresis are obviously improved. Therefore, the improved hysteresis model can increase the prediction accuracy of actual movement.

\subsection{Errors analysis}

The results of various error indicators under different driving signals are shown in Table 1. Based on the actual bending angels and the predicted angles, the maximum absolute error $\Delta_{\max }^{\theta}$ of the static experiment and the dynamic experiment are 1.48 and $1.61^{\circ}$. The maximum relative error $\delta_{\max }^{\theta}$ of the improved hysteresis model is $3.58 \%$. The normalization error $\delta$ of the static experiment and the dynamic experiment are 0.0133 and 0.0162 .

The improved hysteresis model has better prediction with large deformation of the continuous manipulator, while under small tension the error is relatively obvious. Generally speaking, the nonlinear hysteresis model established in this paper can simulate both the desirable tendon displacementbending angle and the undesirable hysteresis loop. The maximum relative error is about $3 \%$, which is mainly due to the asymmetrical hysteresis value generated by the assembly error of two driving tendons. 


\section{Conclusion}

This paper focuses on the hysteresis phenomena of the continuum manipulator which is caused by driving backlash, system friction, tendon elasticity and HNA hysteresis. A hysteresis model is designed based on the Bouc-Wen model, and GA method is utilized to identify the unknown parameters. The static and dynamic experiments show that the improved hysteresis model can predict the actual deformation effectively. The contribution of this paper is building an improved hysteresis model which can describe the positional difference between forward and reverse bending process under the same tendon displacement. This method can also be applied to other similar systems to compensate predicted errors caused by hysteresis. In the future, to increase the hysteresis accuracy, an asymmetric hysteresis model will be used to compensate the errors caused by hysteresis.

Data availability. Data can be made available upon reasonable request. Please contact Wenlong Yang (yangwl@hit.edu.cn).

Competing interests. The authors declare that they have no conflict of interest.

Acknowledgements. This research is supported in part by the National Nature Science Foundation of China under Grant No. 61673139, the State Key Lab Self-planned Project under Grant No. SKLRS201701A, and the Programme of Introducing Talents of Discipline to Universities under Grant No. B07018.

Edited by: Chin-Hsing Kuo

Reviewed by: two anonymous referees

\section{References}

Burgner-Kahrs, J., Rucker, D. C., and Choset, H.: Continuum robots for medical applications: a survey, IEEE T. Robot., 31, 12611280, 2015.

Camarillo, D. B., Milne, C. F., Carlson, C. R., Zinn, M. R., and Salisbury, J. K.: Mechanics modeling of tendon-driven continuum manipulators, IEEE T. Robot., 24, 1262-1273, 2008.

Cruz-Hernandez, J. M. and Hayward, V.: An approach to reduction of hysteresis in smart materials, Proceedings of IEEE International Conference on Robotics and Automation, 2, 1510-1515, 1998.

Du, Z., Yang, W., and Dong, W.: Kinematics modeling and performance optimization of a kinematic-mechanics coupled continuum manipulator, Mechatronics, 31, 196-204, 2015 a.

Du, Z., Yang, W., and Dong, W.: Mechanics modeling of a notched continuum manipulator, J. Mech. Robot., 7, 1114-1125, $2015 \mathrm{~b}$.

Dupont, P. E., Lock, J., Itkowitz, B., and Butler, E.: Design and control of concentric-tube robots, IEEE T. Robot., 26, 209-225, 2010.
Gao, A., Murphy, R., Liu, H., Iordachita, I., and Armand, M.: Mechanical model of dexterous continuum manipulators with compliant joints and tendon/external force interactions, IEEE/ASME T. Mech., 22, 465-475, 2017.

Giorelli, M., Renda, F., Calisti, M., and Arienti, A.: A two dimensional inverse kinetics model of a cable driven manipulator inspired by the octopus arm, Proceedings of IEEE International Conference on Robotics and Automation, 3819-3824, 2012.

Hairer, E., Lubich, C., and Roche, M.: The numerical solution of differential-algebraic systems by Runge-Kutta methods, Springer, Germany, 2006.

Ismail, M., Ikhouane, F., and Rodellar, J.: The hysteresis Bouc-Wen model, a survey, Arch. Comput. Method E., 16, 161-188, 2009.

Jones, B. A., Gray, R. L., and Turlapati, K.: Three dimensional statics for continuum robotics, Proceedings of IEEE/RSJ International Conference on Intelligent Robots and Systems, 26592664, 2009.

Kang, R., Branson, D. T., Zheng, T., Guglielmino, E., and Caldwell, D. G.: Design, modeling and control of a pneumatically actuated manipulator inspired by biological continuum structures, Bioinspir. Biomim., 8, 036008, https://doi.org/10.1088/17483182/8/3/036008, 2013.

Kato, T., Okumura, I., Song, S. E., Golby, A. J., and Hata, N.: Tendon-driven continuum robot for endoscopic surgery: preclinical development and validation of a tension propagation model, IEEE/ASME T. Mech., 20, 2252-2263, 2015.

Kato, T., Okumura, I., Kose, H., Takagi, K., and Hata, N.: Tendondriven continuum robot for neuroendoscopy: validation of extended kinematic mapping for hysteresis operation, Int. J. Comput. Ass. Rad., 11, 569-602, 2016.

Kwok, N. M., Ha, Q. P., Nguyen, M. T., Li, J., and Samali, B.: Bouc-Wen model parameter identification for a MR fluid damper using computationally efficient GA, ISA T., 46, 167-179, 2007.

Li, Z. and Du, R.: Design and analysis of a bio-inspired wire-driven multi-section flexible robot regular paper, Int. J. Adv. Robot. Syst., 10, 209-220, 2013.

Liu, Z., Lai, G., Zhang, Y., Chen, X., and Chen, C. L.: Adaptive neural control for a class of nonlinear time-varying delay systems with unknown hysteresis, IEEE T. Neur. Net. Lear. Syst., 25, 2129-2140, 2014.

Miyasaka, M., Haghighipanah, M., Li, Y., and Hannaford, B.: Hysteresis model of longitudinally loaded cable for cable driven robots and identification of the parameters, Proceedings of IEEE International Conference on Robotics and Automation, 40514057, 2016.

Robinson, G. and Davies, J. B. C.: Continuum robots - a state of the art, Proceedings of IEEE International Conference on Robotics and Automation, 4, 2849-2854, 1999.

Rone, W. S. and Ben-Tzvi, P.: Continuum robot dynamics utilizing the principle of virtual power, IEEE T. Robot., 30, 275-287, 2014.

Rucker, D. C. and Iii, R. J. W.: Statics and dynamics of continuum robots with general tendon routing and external loading, IEEE $\mathrm{T}$ Robot., 27, 1033-1044, 2011.

Ruderman, M., Hoffmann, F., and Bertram, T.: Modeling and identification of elastic robot joints with hysteresis and backlash, IEEE T. Ind. Electron., 56, 3840-3847, 2009.

Seyfferth, W., Maghzal, A. J., and Angeles, J.: Nonlinear modeling and parameter identification of harmonic drive robotic 
transmissions, Proceedings of IEEE International Conference on Robotics and Automation, 3, 3027-3032, 1995.

Simaan, N. and Taylor, R.: A dexterous system for laryngeal surgery multi-backbone bending snakelike slaves for teleoperated dexterous surgical tool manipulation, Proceedings of IEEE International Conference on Robotics and Automation, 351-357, 2004.

Smyth, A. W., Masri, S. F., Kosmatopoulos, E. B., Chassiakos, A. G., and Caughey, T. K.: Development of adaptive modeling techniques for non-linear hysteretic systems, Int. J. NonLinear Mech., 37, 1435-1451, 2002.

Swevers, J., Al-Bender, F., Ganseman, C. G., and Projogo, T.: An integrated friction model structure with improved presliding behavior for accurate friction compensation, IEEE T. Automat. Contr., 45, 675-686, 2000.

Tatlicioglu, E., Walker, I. D., and Dawson, D. M.: New dynamic models for planar extensible continuum robot manipulators, Proceedings of IEEE/RSJ International Conference on Intelligent Robots and Systems, 1485-1490, 2007.

Tavakolpour, A. R., Mat Darus, I. Z., Tokhi, O., and Mailah, M.: Genetic algorithm-based identification of transfer function parameters for a rectangular flexible plate system, Eng. Appl. Artif. Intel., 23, 1388-1397, 2010.

Van Damme, M., Beyl, P., Vanderborght, B., and Van Ham, R.: Modeling hysteresis in pleated pneumatic artificial muscles, Proceedings of IEEE Conference on Robotics, Automation and Mechatronic, 471-476, 2008.
Walker, I. D.: Continuous backbone "continuum" robot manipulators, Isrn Robot., https://doi.org/10.5402/2013/726506, 2013.

Webster, R. J., Okamura, A. M., and Cowan, N. J.: Toward active cannulas: miniature snake-like surgical robots, Proceedings of IEEE/RSJ International Conference on Intelligent Robots and Systems, 2857-2863, 2007.

Wen, Y. K.: Method for random vibration of hysteretic system, J. Eng. Mech. Division, 102, 249-263, 1976.

Wu, L., Song, S., Wu, K., Lim, C. M., and Ren, H.: Development of a compact continuum tubular robotic system for nasopharyngeal biopsy, Med. Biol. Eng. Comput., 55, 403-417, 2017.

Xiao, N. and Guo, S.: Control modeling of a micro-manipulator for human scale tele-operation system, Proceedings of IEEE/RSJ International Conference on Intelligent Robots and Systems, 61256130, 2010.

$\mathrm{Xu}, \mathrm{Q}$.: Identification and compensation of piezoelectric hysteresis without modeling hysteresis inverse, IEEE T. Ind. Electron., 60, 3927-3937, 2013.

York, P. A., Swaney, P. J., Gilbert, H. B., and Rd, W. R.: A wrist for needle-sized surgical robots, Proceedings of IEEE International Conference on Robotics and Automation, 1776-1781, 2015. 